\title{
Glioblastoma Masquerading as a Hypertensive Putaminal Hemorrhage: A Diagnostic Pitfall -Case Report-
}

\author{
Joji INAMASU, Takumi KURAMAE, and Masashi NAKATSUKASA
}

Department of Neurosurgery, Saiseikai Utsunomiya Hospital, Utsunomiya, Tochigi

\begin{abstract}
A 58-year-old man presented with a rare case of glioblastoma masquerading as intracerebral hemorrhage (ICH). He had been medicated for hypertension and diabetes for 10 years before collapsing at home. Brain computed tomography (CT) showed ICH in the right putamen, but CT with contrast medium showed no underlying lesion. He was treated initially with intravenous administration of anti-hypertensive agent under a diagnosis of hypertensive putaminal hemorrhage. ICH aspiration surgery was performed, and serial CT showed ICH resorption. However, he was again admitted for unstable gait and mildly altered mental status 3 months after discharge. Magnetic resonance (MR) imaging with gadolinium showed an enhanced ring-shaped mass around the hematoma cavity. Open biopsy was performed. The histological diagnosis was glioblastoma multiforme, and he was treated with radiation therapy and oral chemotherapy with temozolomide. MR imaging showed marked shrinkage of the tumor, but he died of pneumonia 3 months after the second surgery. In this case, the cause of the hemorrhage was not identified after the seemingly successful hematoma evacuation surgery, and no definitive diagnosis was made until tumor regrowth. Brain tumor should be suspected as a cause of ICH even if the patient has a history of hypertension and the location is typical for hypertensive ICH. Clinical/radiological follow up is essential for detecting subtle neurological deterioration to avoid diagnostic delay.
\end{abstract}

Key words: brain tumor, hypertension, putaminal hemorrhage, intratumoral hemorrhage, diagnosis

\section{Introduction}

Brain tumor is a relatively rare but important cause of intracerebral hemorrhage (ICH). ${ }^{2,4,7)}$ Brain tumor accounted for $4.4 \%$ to $7.2 \%$ of non-traumatic ICHs, ${ }^{6,9)}$ whereas major intratumoral hemorrhage occurred in $1.5 \%$ to $2.9 \%$ of patients with brain tumor. ${ }^{6,9)}$ The incidence of intratumoral hemorrhage depends mainly on the tumor histology and location..$^{2,4,10,11)}$ Intratumoral hemorrhage can usually be identified preoperatively by imaging studies, as the border between the tumor and hemorrhage is usually clear, ${ }^{3,8)}$ but if the hemorrhage is large and expansive enough, the tumor may be compressed and thus not visualized, even if contrast material is used. Therefore, intratumoral hemorrhage may be indistinguishable from spontaneous ICH, and any delay in diagnosis may adversely affect the prognosis for the patient.

We describe a case of glioblastoma masquerading as hypertensive putaminal hemorrhage, which resulted in diagnostic delay.

\section{Case Report}

A 58-year-old man who had been medicated for hypertension and diabetes for 10 years was brought to the emergency department after collapsing at home. He lived alone so

Received January 8, 2009; Accepted March 24, 2009
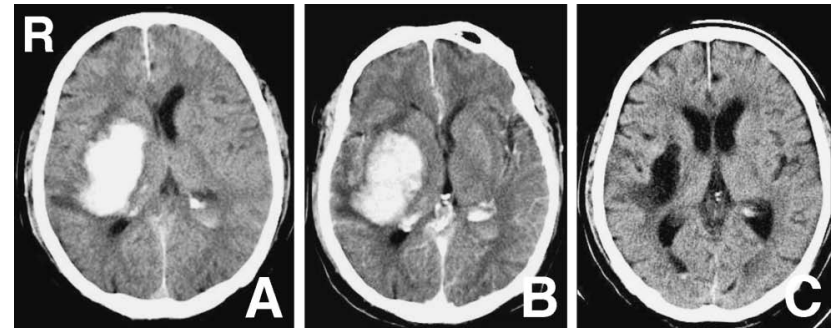

Fig. 1 A: Brain computed tomography (CT) scan showing intracerebral hemorrhage (ICH) in the right putamen. B: CT scan with contrast medium showing no underlying pathology. C: CT scan showing the ICH is resorbed completely at discharge.

the timing was unclear. He showed altered mental status with a Glasgow Coma Scale score of E3V4M5, and left hemiparesis. Blood pressure was 183/111 mmHg. Brain computed tomography (CT) showed ICH in the right putamen (Fig. 1A). CT with contrast medium was performed because of the presence of mild brain edema, but no underlying lesion was identified (Fig. 1B). He was treated initially with intravenous administration of anti-hypertensive agent under a diagnosis of hypertensive putaminal hemorrhage.

ICH aspiration surgery was performed the next day by inserting a drainage tube into the hematoma cavity, with 

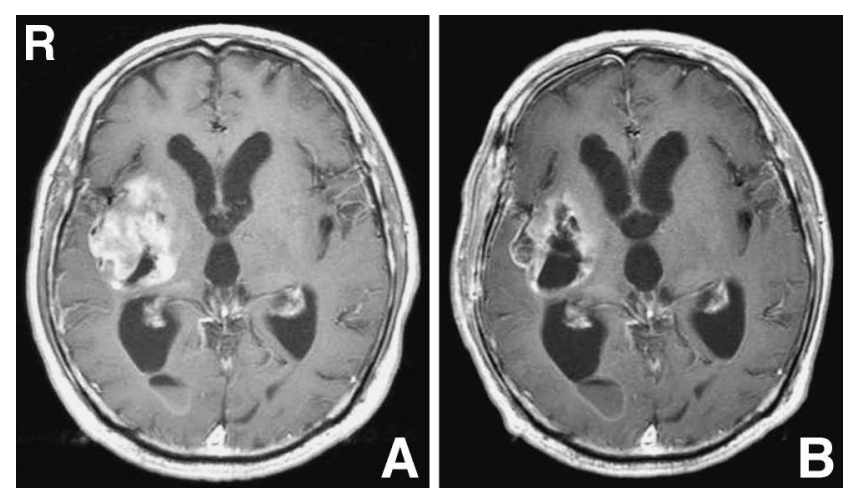

Fig. $2 T_{1}$-weighted magnetic resonance images with gadolinium showing an enhanced mass in the right putamen (A), and marked shrinkage of the tumor after treatment (B).

the use of a Komai stereotactic frame. He made a steady recovery, and serial CT showed ICH resorption (Fig. 1C). He could walk with the aid of brace and cane after rehabilitation, and was transferred to a local rehabilitation facility 40 days after surgery. However, he was referred back to our institution because of unstable gait and mildly altered mental status 3 months after discharge. Magnetic resonance (MR) imaging with gadolinium showed an enhanced ring-shaped mass around the hematoma cavity (Fig. 2A). He was readmitted, and open biopsy was performed. The histological diagnosis was glioblastoma multiforme, and he underwent a combination of radiation therapy and oral chemotherapy with temozolomide. MR imaging showed marked shrinkage of the tumor (Fig. 2B), but he died of pneumonia 3 months after the second surgery. Autopsy was not performed.

\section{Discussion}

In retrospect, our approach to the present case had been biased by the preexisting hypertension. Preoperative CT had demonstrated brain edema around the ICH, an indication of an unusual etiology, ${ }^{7,9)}$ but this was falsely attributed to his delayed admission. Only CT with contrast medium was performed to exclude the presence of underlying pathologies that might cause ICH. Postoperatively, only CT was performed because we were convinced that hypertension was the only cause of the ICH, and also because the surgery was apparently successful.

The present case shows that only preoperative CT with or without contrast medium cannot exclude intratumoral hemorrhage as the cause of ICH, even if the patient has a history of hypertension and the location is typical for hypertensive ICH. As many as $10 \%$ of patients with brain tumor may experience diagnostic delay if CT is the only imaging modality. ${ }^{1)}$ In this respect, MR imaging with gadolinium early in the postoperative period is likely to lead to earlier detection of brain tumor, and should undoubtedly have been performed in this case. However, whether or not every patient with seemingly typical hypertensive ICH needs to undergo MR imaging with gadolini- um to exclude intratumoral hemorrhage is likely to be controversial, particularly from the standpoint of cost effectiveness.

The present case also illustrates the necessity for following up patients with ICH periodically. Early discharge and transfer to rehabilitation facilities is now common to shorten hospital stays. As a result, periodical follow up on an outpatient basis is becoming difficult to achieve, so the opportunity to detect subtle neurological changes in patients with undiagnosed brain tumor may be missed. Therefore, return to the neurosurgery clinic for follow up, or at least transmission of imaging studies from the rehabilitation facilities is important. Efforts to establish closer relationships and exchange of information between neurosurgeons and physiotherapists regarding the progress of rehabilitation in individual patients are also desirable.

Histological examination of the brain parenchyma adjacent to the ICH and the hematoma specimen should also have been performed in this case, which might have led to an earlier diagnosis of brain tumor. The importance of preserving the surgical specimen, fixing in formaldehyde solution, and submission for histological examination soon after hematoma evacuation surgery must be kept in mind. Collecting an ample amount of specimen may be difficult in drainage-oriented stereotactic aspiration surgery despite the minimal invasiveness, so further examination tends to be skipped or even forgotten, as in this case. In contrast, endoscopic hematoma evacuation, also minimally invasive in experienced hands, has the advantage of easily obtaining a surgical specimen during the procedure, and may be a better option compared with stereotactic aspiration surgery. ${ }^{5}$ )

Brain tumor should be suspected as a cause of ICH even if the patient has a history of hypertension and the location is typical for hypertensive ICH. Histological investigation of surgical specimen is essential, even if stereotactic aspiration surgery is performed. Clinical/radiological follow up in the subacute to chronic stage is also essential for detecting subtle neurological deterioration of the patient to avoid diagnostic delay.

\section{References}

1) Bell D, Grant R, Collie D, Walker M, Whittle IR: How well do radiologists diagnose intracerebral tumour histology on CT? Findings from a prospective multicentre study. $\mathrm{Br} \mathrm{J}$ Neurosurg 16: 573-577, 2002

2) Inamasu J, Nakamura Y, Saito R, Kuroshima Y, Mayanagi K, Ichikizaki K: Rebleeding from a primary brain tumor manifesting as intracerebral hemorrhage. Clin Neurol Neurosurg 108: 105-108, 2005

3) Ishikura $\mathrm{R}$, Ando $\mathrm{K}$, Tominaga $\mathrm{S}$, Nakao $\mathrm{N}$, Ikeda J, Takemura Y, Morikawa T: [CT diagnosis of hyperdense intracranial neoplasms: review of the literature]. Nippon Igaku Hoshasen Gakkai Zasshi 59: 105-112, 1999 (Jpn, with Eng abstract)

4) Iwama T, Ohkuma A, Miwa $Y$, Sugimoto S, Itoh T, Takada M, Tanabe Y, Funakoshi T, Sakai N, Yamada H: Brain tumors manifesting as intracranial hemorrhage. Neurol Med Chir (Tokyo) 32: 130-135, 1992 
5) Kim MH, Kim EY, Song JH, Shin KM: Surgical options of hypertensive intracerebral hematoma: stereotactic endoscopic removal versus stereotactic catheter drainage. J Korean Med Sci 13: 533-540, 1998

6) Licata B, Turazzi S: Bleeding cerebral neoplasms with symptomatic hematoma. J Neurosurg Sci 47: 201-210, 2003

7) Morgenstern LB, Frankowski RF: Brain tumor masquerading as stroke. J Neurooncol 44: 47-52, 1999

8) Nakayama Y, Tanaka A, Yoshinaga S, Ueno Y: [Indications for surgery to determine the etiology of subcortical hemorrhage]. No Shinkei Geka 26: 1067-1074, 1998 (Jpn, with Eng abstract)

9) Schrader B, Barth H, Lang EW, Buhl R, Hugo HH, Biederer J, Mehdorn HM: Spontaneous intracranial haematomas caused by neoplasms. Acta Neurochir (Wien) 142: 979-985,
2000

10) Wakai S, Yamakawa K, Manaka S, Takakura K: Spontaneous intracranial hemorrhage caused by brain tumor: its incidence and clinical significance. Neurosurgery 10: 437-444, 1982

11) Yuguang L, Meng L, Shugan Z, Yuquan J, Gang L, Xingang L, Chengyuan W: Intracranial tumoural haemorrhage-a report of 58 cases. J Clin Neurosci 9: 637-639, 2002

Address reprint requests to: Joji Inamasu, M.D., Department of Neurosurgery, Saiseikai Utsunomiya Hospital, 911-1 Takebayashi, Utsunomiya, Tochigi 321-0974, Japan.

e-mail: ginamasu@aol.com 